TITLE:

\title{
Energy exchange in collisions of intrinsic localized modes
}

$\operatorname{AUTHOR}(\mathrm{S})$ :

Doi, Y

CITATION:

Doi, Y. Energy exchange in collisions of intrinsic localized modes.

Physical Review E 2003, 68(6): 066608.

ISSUE DATE:

2003-12

URL:

http://hdl.handle.net/2433/50195

RIGHT:

Copyright 2003 American Physical Society 
PHYSICAL REVIEW E 68, 066608 (2003)

\title{
Energy exchange in collisions of intrinsic localized modes
}

\author{
Yusuke Doi* \\ Department of Aeronautics and Astronautics, Graduate School of Engineering, Kyoto University, Sakyo-ku, Kyoto 606-8501, Japan
}

(Received 27 June 2003; revised manuscript received 29 August 2003; published 18 December 2003)

\begin{abstract}
Energy transfer process is examined numerically for the binary collision of intrinsic localized modes (ILMs) in the Fermi-Pasta-Ulam $\beta$ lattice. Unlike "solitons" in the integrable systems, ILMs exchange their energy in collision due to the discreteness effect. The mechanism of this energy exchange is examined in detail, and it is shown that the phase difference is the most dominant factor in the energy exchange process and, generally speaking, the ILM with advanced phase absorbs energy from the other. Heuristic model equations which describe the energy transfer of ILMs are proposed by considering the ILMs as interacting "particles." The results due to these equations agree qualitatively very well with those of the numerical simulations. In some cases, the relation between the phase difference of the ILMs and the transferred energy becomes singular, which may be regarded as one of the major mechanisms responsible for the generation of "chaotic breathers."
\end{abstract}

DOI: 10.1103/PhysRevE.68.066608

PACS number(s): 05.45.Yv, 63.20.Pw, 63.20.Ry

\section{INTRODUCTION}

Since the first report by Sievers and Takeno [1], the intrinsic localized mode (ILM) has been studied extensively by many researchers not only numerically but also analytically [2]. The ILM is a localized excitation which appears in nonlinear discrete systems in various fields of physics such as material science, optics, and electrical engineering [3,4]. It is excited by a balance between nonlinearity and discreteness, and its frequency is located out of the linear dispersion band. Unlike the impurity localized mode being fixed on impurities, ILMs can appear at arbitrary position of the system. Adding to these stationary ILMs, it is expected that moving ILMs such as soliton exist [5]. But a moving ILM, in general, loses its energy as it propagates in the system due to the so-called Peierls-Nabarro (PN) potential which results from the discreteness of the system [6]. Moving ILMs can be stopped by this potential barrier when the discreteness is large enough [7]. Exact solutions of moving ILMs have not been found, but long-lived moving ILMs appear in various numerical calculations with Fermi-Pasta-Ulam (FPU) lattice, because the PN potential in the FPU lattice is relatively small. This fact indicates an apparent stability of a single moving ILM in the FPU system. It is natural to question what happens in the collision of moving ILMs. However, interaction (or collision) processes of moving ILMs have not yet been clarified and still remain an interesting problem to be studied.

It is well known that long-wavelength phenomena in lattice systems are described approximately by evolution equations in the continuum framework. Although the displacement of particles of the ILM is optical-mode-like, that is, $u_{n}=(-1)^{n} a_{n}$, its envelope $a_{n}$ can be described by the nonlinear Schrödinger (NLS) type equation. Solitons in integrable systems such as the NLS equation do not suffer amplitude changes in the collisions, but those in the nonintegrable systems affect each other by collision. Recently, Dmitriev et al. reported inelastic collisions of solitons

\footnotetext{
*Electronic address: yusuke_doi@kuaero.kyoto-u.ac.jp
}

in perturbed NLS equations [8-10]. They examined the change of velocity of two solitons numerically. They found that the solitons exchange their energies, and this exchange depends on the phase difference before the collision. In the equal energy (amplitude) case, the change of velocity with respect to the phase difference $\Delta \Phi$ becomes irregular near $\Delta \Phi=0$. On the other hand, the velocity does not change (therefore elastic collision) in the case of $\Delta \Phi=\pi$. In the region of the irregular change, the fractal pattern in the velocity change was observed, which is due to the short-lived two-soliton bound state. Also, the discreteness affects the behavior of solitons in collision process. For example, Cai et al. reported that there are two states which interlacingly appear depending on the incoming velocity before the collision, that is, the bound state and the escape [11]. These two states appear not only in the case of equal amplitude with symmetry, but also in the case of different amplitude. To investigate collision processes of ILMs, on which we focus in this paper, will be an interesting problem because the discreteness can lead to behaviors differing from continuous solitons.

One of the characteristic properties of ILMs due to their discreteness is the symmetry difference. Unlike solitons, there are two modes possible for a stationary ILM, one with its peak of amplitude on the particle (Sievers-Takeno mode) [1] and the other on the center of the two particle (Page mode) [12]. Moving ILMs can take intermediate mode while they progress from particle to particle. It is expected that the collision of ILMs is more complex than that of usual soliton, because of this symmetry difference.

Chaotic breathers (CBs) might be one of the most interesting phenomena in the dynamics of the ILMs in lattice systems [13-16]. The modulational instability from the highest frequency mode ( $\pi$ mode) generates a number of ILMs and their collisions lead to a large amplitude single ILM with an extreme energy localization. Many ILMs excited by the modulational instability collide with each other randomly and exchange their energy. The number of the ILMs decreases and the amplitudes of remaining ILMs become larger in time, then only one ILM with large amplitude survives and propagates in an erratic way (in this sense this single 
ILM is called chaotic breather) and it gathers about $90 \%$ of the whole energy of the system. Finally, this ILM collapses and the system goes to an equipartition state in which energy is transferred to lower frequency modes.

The mechanism has also been proposed to explain why only one ILM grows through the collisional process. If the ILM which has larger energy absorbs energy of smaller ILM, after many successive collisions, the ILM which has the largest energy comes to get most energy of the system. This explanation is supported by some numerical simulations in which a larger ILM is set to collide with three small ILMs (also in Ref. [13]). The statistical analysis of the transferred energy in the collisional process in the Klein-Gordon system has shown in Refs. $[17,18]$ that the larger ILMs prefer to become larger after collision in almost all cases.

The purpose of this paper is to clarify detailed mechanisms of energy transfer between ILMs in the binary collision. To do so, we examine the collision of two ILMs and we discuss the relation between the ILM's growth in the CB formation process and the energy transfer in collision of two ILMs.

This paper is organized as follows. In Sec. II, the model that we consider is explained. In Sec. III, the numerical method and the definition of parameters are presented. In Sec. IV, results of the numerical simulation of the collision of ILMs are shown, classifying into three cases: (a) same energy, (b) small energy difference, and (c) large energy difference. In Sec. V, the mechanisms of energy transfer are discussed by proposing model equations to compare with numerical results in Sec. IV. The mechanisms of energy transfer are also discussed in relation to CBs. Concluding remarks are given in Sec. VI.

\section{THE MODEL}

We consider the one-dimensional Fermi-Pasta-Ulam $\beta$ (FPU $\beta$ ) chain of particles with unit mass. Each particle interacts only with its nearest-neighbor particles. The Hamiltonian of the system is given by

$$
H=\sum_{n}\left[\frac{\dot{u}_{n}^{2}}{2}+\frac{\alpha}{2}\left(u_{n+1}-u_{n}\right)^{2}+\frac{\beta}{4}\left(u_{n+1}-u_{n}\right)^{4}\right],
$$

where $u_{n}$ represents the displacement of the $n$th particle from equilibrium point, $\alpha$ and $\beta$ represent harmonic and anharmonic coefficients of interparticle reaction, respectively. The equations of motion can be derived from Eq. (1) to yield

$$
\begin{aligned}
\ddot{u}_{n}= & \alpha\left(u_{n-1}-2 u_{n}+u_{n+1}\right)+\beta\left(u_{n-1}-u_{n}\right)^{3} \\
& +\beta\left(u_{n+1}-u_{n}\right)^{3} .
\end{aligned}
$$

In this paper we set $\alpha=1$ and $\beta=4$ in all the numerical simulations. Note that the system considered here is the $k_{2}-k_{4}$ system for which the ILM was first studied by Sievers and Takeno [1]. Since ILMs in this system have basic and important properties of ILMs, we investigate, in this paper, the collisional process based on this system. Adding to this, we examine the collision of ILMs only in the case of hard potential. We do not consider the case of soft potential $\left(k_{4}\right.$
$<0)$ but ILMs do not exist in the FPU system with soft potential. Therefore, our results can be generic at least within the FPU $\beta$ system.

\section{NUMERICAL SIMULATION}

The ILMs that we use in simulations are generated by a preliminary calculation. In this calculation, the following disturbance is added to the system as initial conditions:

$$
\begin{gathered}
u_{N / 2-1}=-\frac{a_{0}}{2}, \quad \dot{u}_{(N / 2)-1}=w, \\
u_{N / 2}=a_{0}, \quad \dot{u}_{N / 2}=0, \\
u_{N / 2+1}=-\frac{a_{0}}{2}, \quad \dot{u}_{N / 2+1}=-w, \\
u_{i}=0, \quad \dot{u}_{i}=0 \quad(i \text { otherwise }) .
\end{gathered}
$$

After some time evolution, moving ILMs are excited depending on the amplitude $a_{0}$ and kicking parameter $w$. We extract them as numerical data and stock them to use for later simulations. The relation between energy and frequency of ILMs is shown in Fig. 1(a), and that between energy and width is also shown in Fig. 1(b). We can see that these ILMs can be arranged in one family.

Here, we define energy $E$ and region $R$ of ILMs. First, the energy of each particle, $e_{n}$, is defined as

$$
e_{n}=\frac{1}{2} u_{n}^{-2}+\frac{1}{2}\left(v_{n-1}+v_{n}\right),
$$

where $v_{n-1}$ and $v_{n}$ are potential energies between the $n$th particle and its nearest-neighbor particles on both sides given as

$$
v_{n}=\frac{\alpha}{2}\left(u_{n+1}-u_{n}\right)^{2}+\frac{\beta}{4}\left(u_{n+1}-u_{n}\right)^{4} .
$$

We find the maximum particle energy of the ILM, say $e_{\max }$. The region $R$ of the ILM is defined as the range of particles around $e_{\max }$ whose particle energy is larger than some critical value $e_{b}$. Then the energy $E$ of the ILM is the total energy of the particles in such a region given as

$$
\begin{gathered}
E=\sum_{n \in R} e_{n}, \\
R=\left\{n \mid e_{n}>e_{b}\right\} .
\end{gathered}
$$

We choose $1 \%$ of the maximum particle energy $e_{\max }$ as $e_{b}$ to determine the region $R$, that is,

$$
e_{b}=0.01 e_{\max } .
$$

Note that this value can vary in time and affects the width and the energy of the ILM, since $e_{\max }$ can vary in time. From this point of view, this ratio is preferred to be smaller. But if the ratio is too small, $e_{b}$ becomes smaller than the energy of the (linear) ripples and the width of ILM becomes extraordi- 

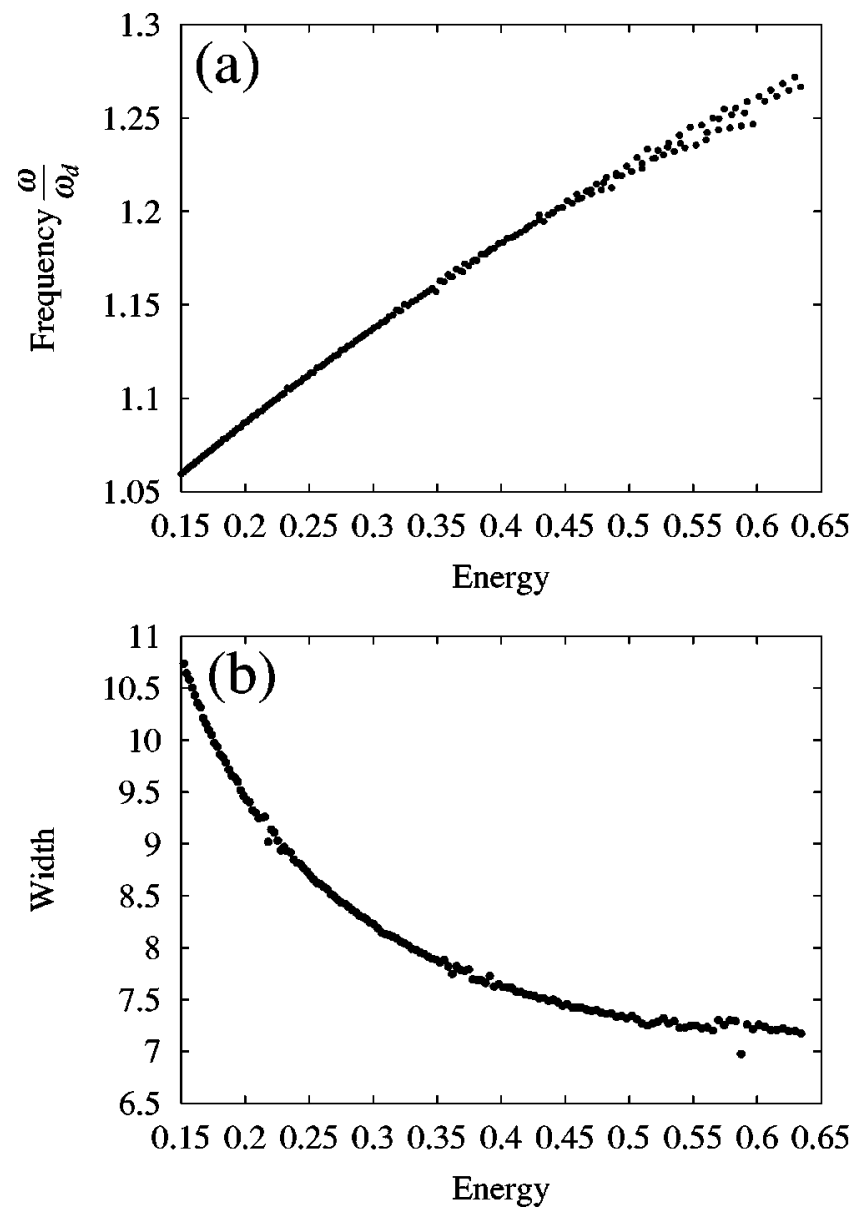

FIG. 1. Relation between ILMs' energy and (a) frequency and (b) width, where $\omega_{d}$ denotes the highest frequency of the linear dispersion band ( $\pi$ mode) .

nary long. The choice of $1 \%$ critical value is found to be a suitable one in subsequent calculations.

We now define the phase of ILMs. In contrast to usual solitons, each particle in ILMs vibrates in opposite phases with respect to the nearest-neighbor particles. To remove such opposite displacements, for convenience, we introduce the following variable transformation:

$$
\bar{u}_{n}=(-1)^{n} u_{n} .
$$

Then we define the phase $\phi_{n}$ of the $n$th particle as

$$
\phi_{n}=\tan ^{-1}\left(\frac{\dot{\bar{u}}_{n}}{\bar{u}_{n}}\right) .
$$

In the case of stationary ILM, $\phi_{n}$ 's of the particles in the ILM take perfectly the same value. On the other hand, in the case of moving ILM, $\phi_{n}$ 's are not perfectly the same, but deviate from the phase of the center particle of the ILM. However, deviations are small, i.e.,

$$
\max \left\{\left|\phi_{n}-\phi_{\text {center }}\right|, n \in R\right\} \ll 2 \pi \text {. }
$$

So we can regard the phase of the ILM, $\Phi$, as that of the center particle of the ILM.

Next, we show the way of the simulation. Initially, two ILMs separated at some distance are introduced into the system so that they approach. Hereafter, the ILM introduced on the left side is called mode $M_{1}$ and that on the right side mode $M_{2}$. Other parameters such as energy $E$, phase $\Phi$, etc., are also labeled by the same rule. Initial amplitudes of the ILMs are varied from small to large and phase are also changed from 0 to $2 \pi$ at each set of $E_{1}, E_{2}$. We pursue the ILMs colliding and interacting until they are separated by enough distance. Various phases are taken as ILM's initial condition, so that the phase difference of two ILMs when they collide can change from 0 to $2 \pi$. We examine temporal evolutions of ILMs' position, amplitude (energy), frequency, and phase in the whole time of calculations. The sixth-order symplectic integration method was used for numerical integration of Eq. (2).

Note that the collision point of the ILMs can be changed depending on the initial conditions, so that it is not restricted on-site or intersite. Because ILMs change their shapes and symmetry as they propagate in the system, we would get the result for any collision point by calculating with various initial conditions. No significant difference was seen from the view of difference of collision point.

\section{NUMERICAL RESULTS}

Numerical results are shown by classifying into three cases depending on the energy differences in two ILMs, i.e., (a) same energy, (b) small energy difference, and (c) large energy difference. The results are given as follows.

\section{A. Same energy case}

We examine the collision dynamics of ILMs which have the same energy $E_{1}=E_{2}$. First, we show the result in the case that two ILMs have the same phase $\Phi_{1}=\Phi_{2}$. In this case, two ILMs fuse at an instance of collision. However, behaviors of ILMs after fusion drastically differ depending on whether two ILMs are antisymmetric (mirror symmetric) or not.

Figure 2 shows these dynamics as the position of ILMs and energy intensity contour of each particle. In the antisymmetric case [Fig. 2(a)], two ILMs continue to interact and form a kind of bound state. That is, two ILMs repeat the state of fusion and the state of separation. Adding to this, ILMs do not move from the position where they collide. This state seems to be a bound state of ILMs similar to the one reported in Ref. [19].

In other cases, two ILMs are separated away after fusion. Figure 2(b) shows one of those cases. After the collision, the energies of the ILMs are no longer the same $\left(E_{1} \neq E_{2}\right)$. Two ILMs exchange their energies while they collide. Therefore the velocity, frequency, and phase of two ILMs become different from those before the collision.

Second, we show the case of two ILMs with different phases $\left(\Phi_{1} \neq \Phi_{2}\right)$. Here we define the phase difference $\Delta \Phi$ as 

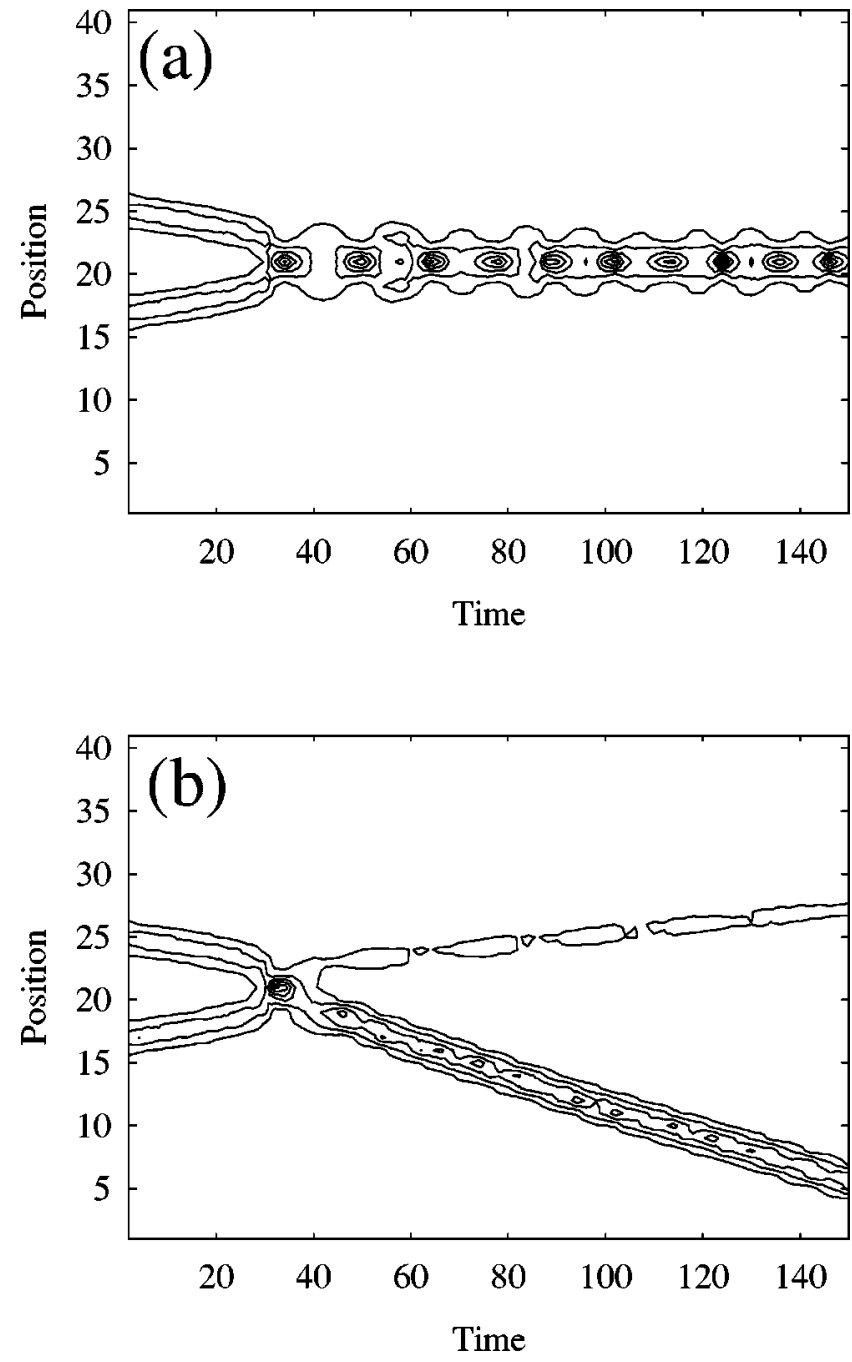

FIG. 2. Energy contour for the collision of two ILMs. Temporal changes of ILMs' energy and position are shown. Energies of two ILMs are the same $\left(E_{1}=E_{2}=0.60\right)$ and the phase difference $\Delta \Phi$ $=0$ at the instance of collision; (a) mirror-symmetric case and (b) asymmetric case.

$$
\Delta \Phi=\Phi_{2}-\Phi_{1}
$$

The range of $\Delta \Phi$ is $-\pi / 2<\Delta \Phi<\pi / 2$. When $\Delta \Phi>0$, the phase of the right ILM $\left(\Phi_{2}\right)$ is advanced and when $\Delta \Phi$ $<0$, it is delayed. We change the initial phase of one ILM from 0 to $2 \pi$ by $2 \pi / 200$. The energy of ILM, $E$ ( $=E_{1}$ $=E_{2}$ ), is varied from 0.20 to 0.60 by 0.05 . Here we show three results for the cases (1) $\Delta \Phi=\pi / 2$, (2) $\Delta \Phi=-\pi / 2$, and (3) $\Delta \Phi=\pi$, with $E=0.60$.

The result in the case of $\Delta \Phi=\pi / 2$ is shown in Fig. 3(a). In collisional process, $M_{1}$ gives some energy to $M_{2}$. After the interaction, two ILMs are repelled away. As a result of energy transfer, frequency and velocity of the two ILMs change to other values. Then the phase difference $\Delta \Phi$ changes in time at a constant rate.

In the case of $\Delta \Phi=-\pi / 2$, being contrary to the case of $\Delta \Phi=+\pi / 2, M_{2}$ gives some energy to $M_{1}$. The changes of frequency and velocity are just contrary to the case of $\Delta \Phi$ $=\pi / 2$. These behaviors are shown in Fig. 3(b).
In the case of $\Delta \Phi=\pi$, that is, two ILMs are just out of phase, they do not exchange energies. Even after collision, energy of ILMs are $E_{1}^{\prime}=E_{2}^{\prime}=0.60$ as is shown in Fig. 3(c). (Here and hereafter, a prime denotes a quantity after the collision.) Therefore the frequencies of ILMs do not change and the phase difference is kept as $\Delta \Phi=\pi$.

Figure 4 shows the time evolution of the position and the energy contour of particles of the ILMs corresponding to the three cases shown in Fig. 3. Unlike the case of $\Delta \Phi=0$ shown in Fig. 2, the ILMs transfer energy and are repelled without merging.

We examine the relation between the energy of the ILMs after collision and the phase difference at the collision. Figure 5 shows such a relation for the case $E_{1}=E_{2}=0.60$. We can see that the magnitude of transferred energy depends on the phase difference of the ILMs. The ILMs exchange their energies maximum at $\Delta \Phi=0$ (except for perfectly antisymmetric case as seen above), and do not exchange the energies at $\Delta \Phi=\pi$. When $\Delta \Phi>0, E_{2}^{\prime}$ becomes larger than $E_{1}^{\prime}$, and when $\Delta \Phi<0, E_{1}^{\prime}$ becomes larger than $E_{2}^{\prime}$. From this result, we conclude that the ILM with advanced phase absorbs energy of the other ILM through collisional process.

Lastly, we check the relation of the transferred energy to the ILM's energy. Even if the ILM's energy $E=E_{1}=E_{2}$ is changed, the dependence of the transferred energy on the phase difference $\Delta \Phi$ is not changed. The ratio of the maximum transferred energy (termed hereafter the energy exchange ratio) at $\Delta \Phi=0$, i.e.,

$$
\Delta \bar{E}^{\prime}=\frac{\left|E_{2}^{\prime}-E_{1}^{\prime}\right|}{E_{1}^{\prime}+E_{2}^{\prime}}
$$

with respect to the phase difference is shown in Fig. 6 for different values of the ILM's energy $E$. In the case with small $E$, the energy exchange ratio $\Delta \bar{E}^{\prime}$ increases as ILM's energy increases. However this variation becomes smaller as $E$ becomes larger. The difference of $\Delta \bar{E}^{\prime}$ due to the magnitude of $E$ is much smaller than that due to the phase difference. Therefore the phase difference of the ILM causes the most dominant effect on the energy exchange.

Figure 6 shows that, in a region around $\Delta \Phi=0$, the energy exchange ratio varies irregularly and the difference of $\Delta \bar{E}^{\prime}$ due to the magnitude of $E$ becomes larger than in the other region. We examine the behaviors of ILMs in such a region in a sequel. It should be pointed out here that the dependence of transferred energy on phase difference in a perturbed NLS system is considered in Ref. [8], and also an irregular behavior is found near $\Delta \Phi=0$. This property seems to be caused by the same mechanism. Resonance of solitons, which forms a bound state of solitons, is also reported in Ref. [11] but there is some difference from that in the FPU system, that is, the behavior of ILMs might change when symmetry of ILMs differs.

\section{B. Small energy difference case}

We show the result in the case where $E_{1} \neq E_{2}$ and $\Delta \bar{E}$ $=\left|E_{1}-E_{2}\right| /\left(E_{1}+E_{2}\right)$ is relatively small. Note that frequen- 

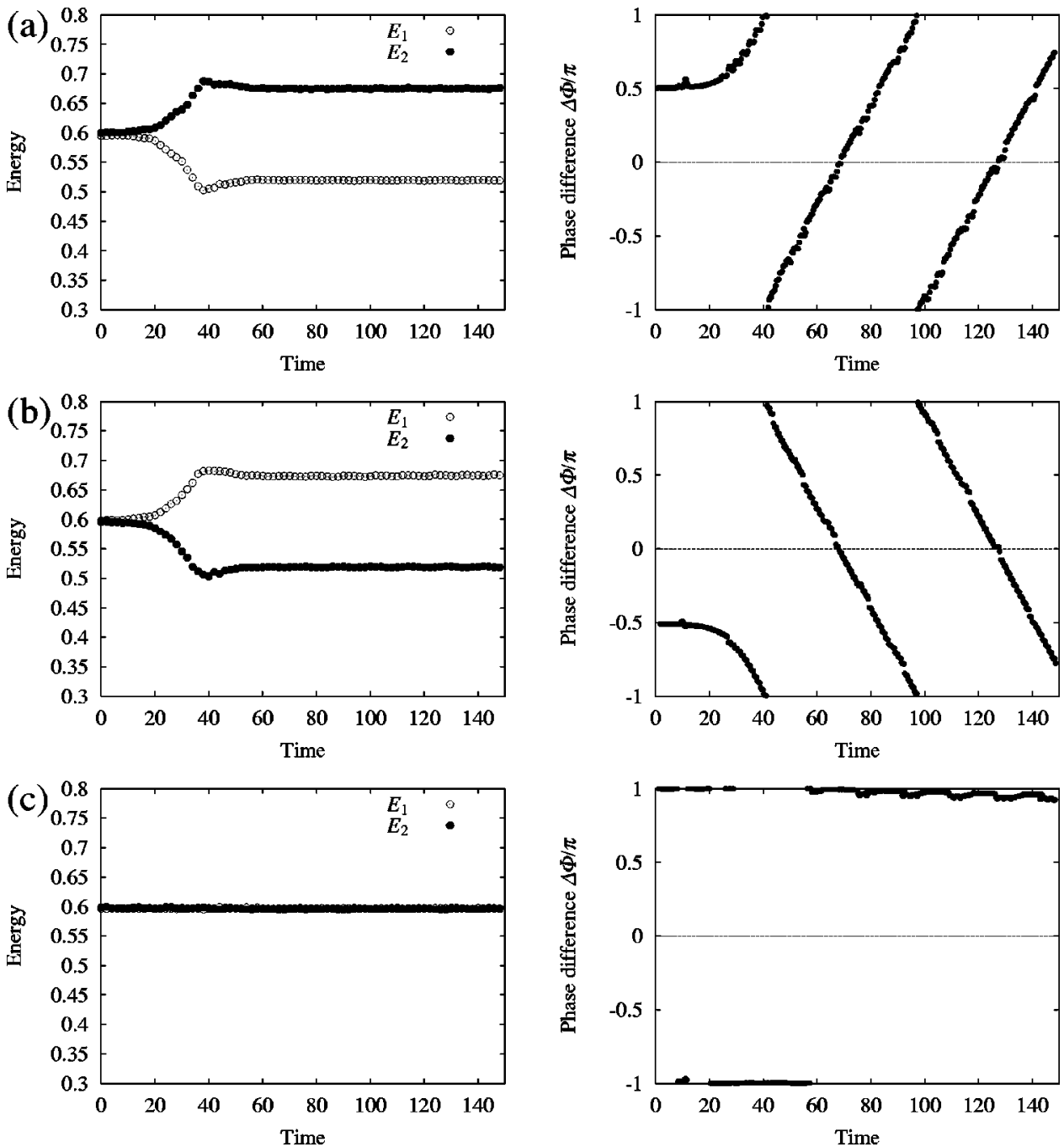

FIG. 3. Dynamics of two ILMs during collision. Left column represents temporal change of the energy of ILMs, $E_{1}, E_{2}$, and right column represents that of the phase difference, $\Delta \Phi:$ (a) $\Delta \Phi=\pi / 2$, (b) $\Delta \Phi=-\pi / 2$, and (c) $\Delta \Phi=\pi$. cies the ILMs are different at $t=0$, because of $E_{1} \neq E_{2}$. The phase difference of the ILMs may change in time even before collision. That is why we can not determine exactly the phase difference at the very moment of the collision. Here we regard the phase difference as the value of $\Delta \Phi$ at $t=0$.

We show four typical results in the case of $E_{1}=0.20$ and $E_{2}=0.25$ in Fig. 7. Differences in these results depend on the amount and sign of the phase difference $\Delta \Phi$ of the ILMs at the moment of the collision: (a) $\pi / 2<\Delta \Phi<\pi$, (b) $0<\Delta \Phi$ $<\pi / 2$, (c) $-\pi<\Delta \Phi<-\pi / 2$, and (d) $-\pi / 2<\Delta \Phi<0$. In each figure, left column shows the time evolution of the ILMs' energies $E_{1}$ and $E_{2}$, right column shows the change of the phase difference, $\Delta \Phi$, with time.

In the case (a) [Fig. 7(a)], the ILMs begin to interact when the phase of $M_{2}$ is advanced $(\Delta \Phi>0)$ at $t=30$. As we see in the case of the same energy, $M_{2}$ absorbs energy from $M_{1}$. The phase difference $\Delta \Phi$ spreads until $\Delta \Phi=\pi$, because of $E_{2}>E_{1}$. The sign of $\Delta \Phi$ is reversed when $\Delta \Phi$ exceeds $\pi$ $(t=62)$. Now the phase of $M_{2}$ is behind that of $M_{1}$, so that the energy of $M_{2}$ is absorbed by $M_{1}$. When the ILMs are apart enough not to interact $(t=140)$, the energy becomes the same $\left(E_{1}^{\prime}=E_{2}^{\prime}=0.225\right)$ and the phase difference becomes constant in time.
In the case (b), the energy which $M_{2}$ absorbs from $M_{1}$ while $\Delta \Phi>0$ is larger than that in the case (a). This is because the time duration from $t=40$ to $t=72$ in which the phase of $M_{2}$ is advanced is longer than the case (a). When $t=72, M_{2}$ gets the energy of 0.09 from $M_{1}$ which is about six times larger than the case (a). After $\Delta \Phi$ becomes negative, $M_{2}$ loses its energy. When the ILMs do not interact $(t$ $=83$ ), energies of the ILMs are $E_{1}^{\prime}=0.17$ and $E_{2}^{\prime}=0.28$. As a result, the energy of 0.3 moves from $M_{1}$ to $M_{2}$ in the collisional process.

In the case (c) [Fig. 7(c)], the ILM's interaction begins when the phase difference is almost $\pi$, and the interaction progresses in the stage when the phase of $M_{2}$ is in delay $(\Delta \Phi<0) . M_{2}$ loses its energy and then, at $t=75$, the size of the ILMs energy is interchanged. Therefore the ratio of the change of phase difference is also reversed. After the change in direction of energy transfer due to the change in the sign of $\Delta \Phi$, the interaction of the ILMs ends at about $t=130$. The energies after collision become $E_{1}^{\prime}=0.255$ and $E_{2}^{\prime}$ $=0.195$ and, as a result, the energy of 0.055 transfers from $M_{2}$ to $M_{1}$.

Finally, in the case (d) [Fig. 7(d)], the ILMs begin to interact when the phase difference is $\Delta \Phi=-\pi / 2(t=35)$. 

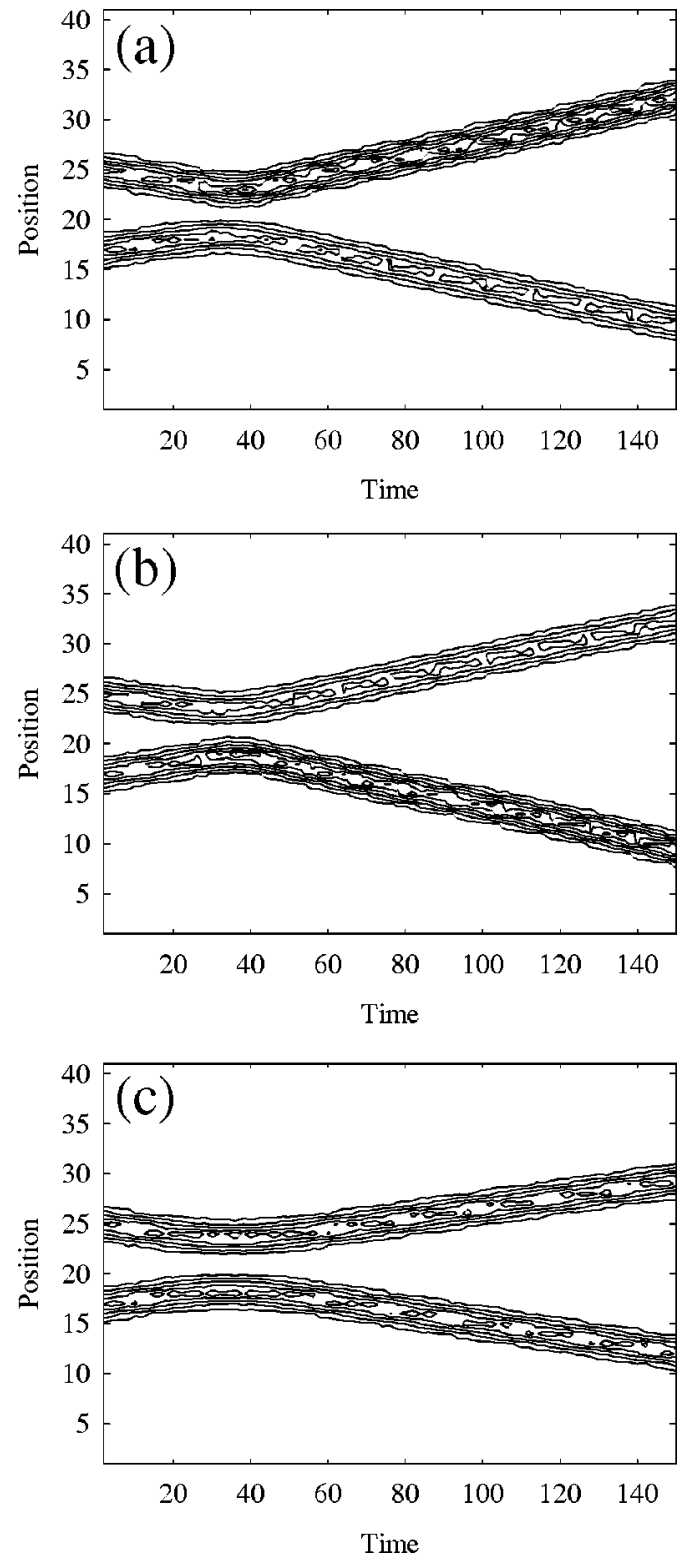

FIG. 4. Energy contour for the collision of two ILMs. Same as Fig. 2 except for $\Delta \Phi \neq 0$. Each figure corresponds to that in Fig. 3.

Interaction progresses in the same stage as in the third case, when the phase of $M_{2}$ is in delay, but the phase difference approaches more closer to 0 than that in the case (c). Therefore more energy is transferred from $M_{2}$ to $M_{1}$ in this first stage of collision because of the same reason mentioned in the case (b). Directions of the energy transfer are reversed due to the reversion of the sign of $\Delta \Phi$ at $t=81$ and the collisional process terminates at $t=93$ with $E_{1}^{\prime}=0.3$ and $E_{2}^{\prime}$ $=0.15$. As a result the energy of 0.1 moves from $M_{2}$ to $M_{1}$, which is about two times larger than that in the case (c).

Figure 8 shows the temporal changes of ILM's position exhibited by energy intensity contours. It can be seen that ILMs significantly affect each other when they approach in the case (b) and case (d). Therefore this may be the reason for large energy transfer in these cases compared with the other cases.

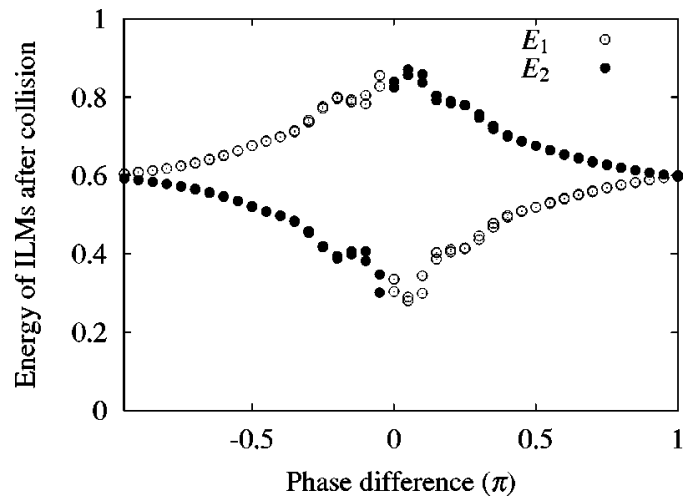

FIG. 5. Relation between phase difference at the instance of collision $(\Delta \Phi)$ and the energies of ILMs after collision $\left(E_{1}^{\prime}\right.$ and $\left.E_{2}^{\prime}\right)$. Phase difference is normalized by $\pi$.

We can summarize some differences in results between the case of the same energy and the case of small energy difference as follows.

(1) In the case of small energy difference, $\Delta \Phi$ changes even before the collision because of the energy difference of two ILMs.

(2) Due to this nonzero change of the phase difference $d \Delta \Phi / d t$ before the collision (for example, $0.082 \times 2 \pi \mathrm{rad} / \mathrm{s}$ for the case of $E_{1}=0.25, E_{2}=0.25$ ), the reversion of the sign of $\Delta \Phi$ can occur.

(3) Direction of energy transfer depends on the sign of the phase difference. Small energy difference can lead to the reversing of energy amount of ILMs. This reversion also causes the change of the sign of $d \Delta \Phi / d t$.

These factors lead to more complicated dynamics. In view of the relation of the phase difference to the transferred energy (Fig. 9), we find the following differences which may be due to the temporal change of the phase difference.

(a) In the case of $E_{1}=E_{2}$, we can regard the peak of the transferred energy as single peak $(\Delta \Phi=0)$. Whereas there exist two peaks which belong to either $E_{1}>E_{2}$ or $E_{1}<E_{2}$.

(b) In the case with the same energy, the curve with respect to $\Delta \Phi$ and $E_{1}, E_{2}$ is symmetric about the point $\Delta \Phi$ $=\pi$, but this symmetry is lost at any point in the case of $E_{1} \neq E_{2}$.

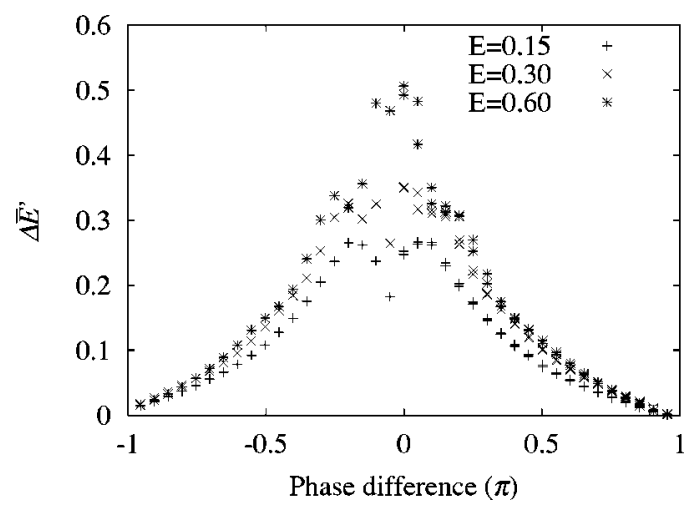

FIG. 6. Relation between the phase difference and the energy exchange ratio $\Delta \bar{E}^{\prime}$. 

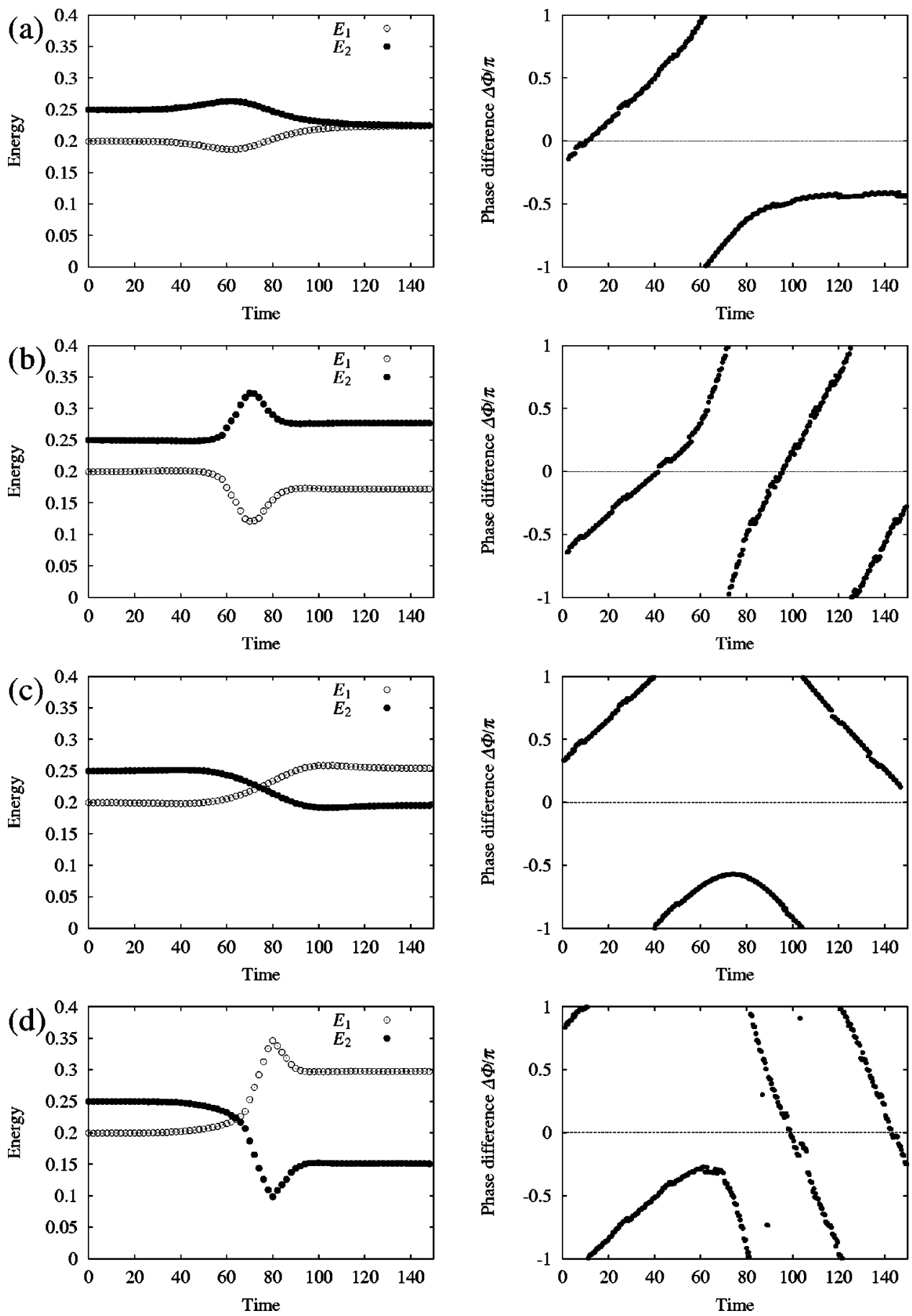

FIG. 7. Dynamics of two ILMs during collision. Same as Fig. 3 except for $E_{1}=0.20, E_{2}$ $=0.25$. Four typical cases which may depend on the phase difference at the instance of collision are shown: (a) $\pi / 2<\Delta \Phi<\pi$, (b) $0<\Delta \Phi<\pi / 2, \quad$ (c) $\quad-\pi<\Delta \Phi$ $<-\pi / 2$, and (d) $-\pi / 2<\Delta \Phi$ $<0$.
We can see a discontinuous change of the energy near $\Delta \Phi=-\pi / 2$ in Fig. 9. This change seems to be equivalent to the irregular change of the transferred energy near $\Delta \Phi=0$ in the same energy case (see Figs. 5 and 6). We examine the dynamics of the ILMs in this region in detail. One of the results is shown in Fig. 10. In this case, the ILMs react more strongly than in other cases, that is, they once fuse and are separated, and then they attract each other leading to recollision. This behavior appears to be sensitive to $\Delta \Phi$ at the beginning of collision. We would be dealing with this point in detail in the following section.

\section{Large energy difference case}

When the difference of the energy is large, the behavior of the ILMs is quite different from that shown above. We show a result for the case of $E_{1}=0.20$ and $E_{2}=0.60$ in Fig. 11 . The change of the ILM's position with time is also shown in Fig. 12.

We compare these results with those in other cases to arrive at the following items.

(1) For the large energy difference case, $\Delta \Phi$ changes in time before the collision similarly as in the case of small energy. 

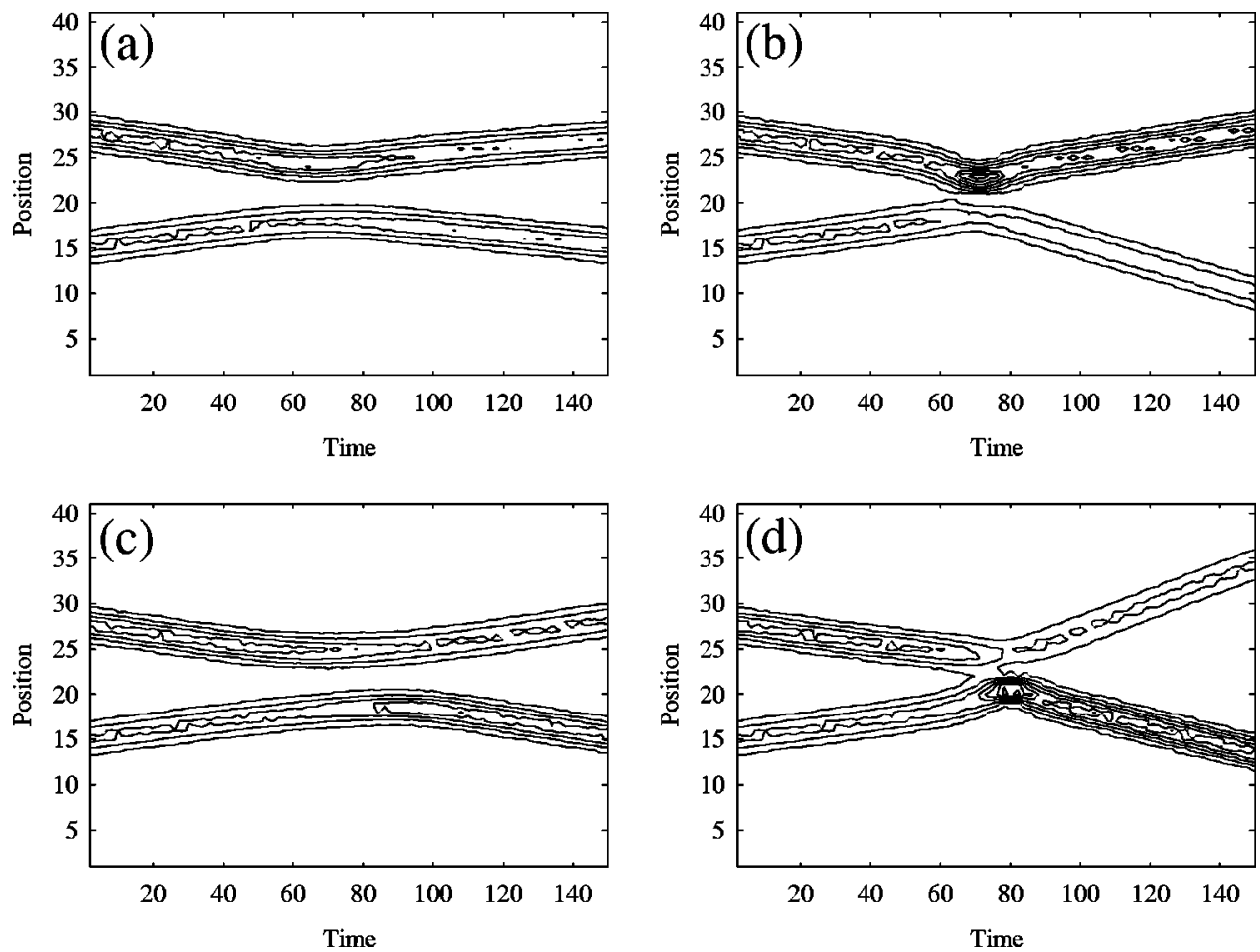

FIG. 8. Energy contour for the collision of two ILMs. Same as Fig. 4 except for $E_{1}=0.20, E_{2}$ $=0.25$. Each figure corresponds to that in Fig. 7.

(2) However, the change of the phase difference is faster than the case of small energy (for example, $d \Delta \Phi / d t$ $=0.0541 \times 2 \pi \mathrm{rad} / \mathrm{s}$ for the case of $E_{1}=0.20, E_{2}=0.60$ ). Therefore the reversing of the phase advance can take place more often than the case of small energy difference, and each time duration in which energy transfers to one direction becomes shorter than the case of small energy.

(3) Therefore, reversings of the direction of energy transfer take place more often than the small energy case. But interchange of ILM's energy does not occur because the energy difference is large and the transferred energy in each stage is small due to its shorter time duration.

Therefore, in the case of large energy difference, there is no significant difference in temporal energy changes among

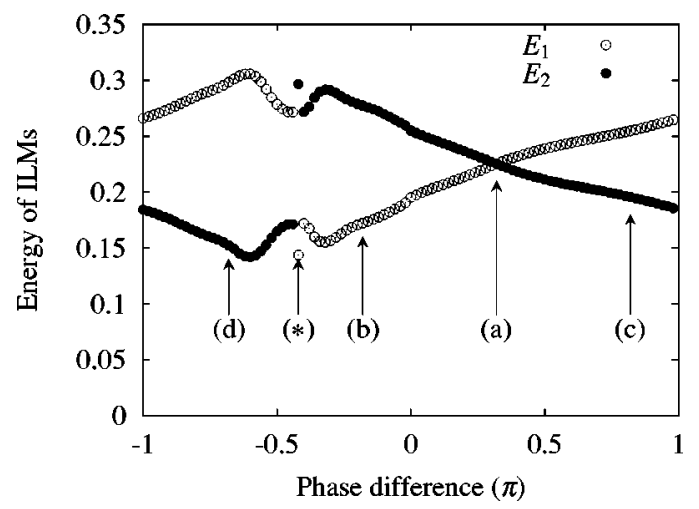

FIG. 9. Relation between the phase difference $\Delta \Phi$ at the instance of collision and energy of ILMs after collision. Same as Fig. 5 except for $E_{1}=0.20, E_{2}=0.25$. Labels (a) $-($ d) denote the phase difference corresponding to the cases (a)-(d) in Fig. 7 and label (*) denotes phase difference where multiple collision occurs (corresponding to Fig. 10). the results for different values of the initial phase difference. Note that the maximum energy which is exchanged in this process is about 0.1 , but the resultant change of energy after collision is much smaller than 0.1 in almost all the case owing to repeated reversings of the direction.

The relation of the phase difference to the transferred energy is shown in Fig. 13. Comparing with other cases, there are some differences, given as follows.

(1) Dependence of the energy on the phase difference shows that the transferred energy is nearly zero in many cases, but a larger ILM becomes larger in many cases. Adding to this, reversion of the magnitude of ILM's energy does not take place.

(2) Figure 13 shows that there exist many peaks in the transferred energy according to the change in the phase difference.

Figure 14 shows ranges of ILMs' energy after collision with fixed $E_{1}(=0.3)$ and various $E_{2}$. We can classify the

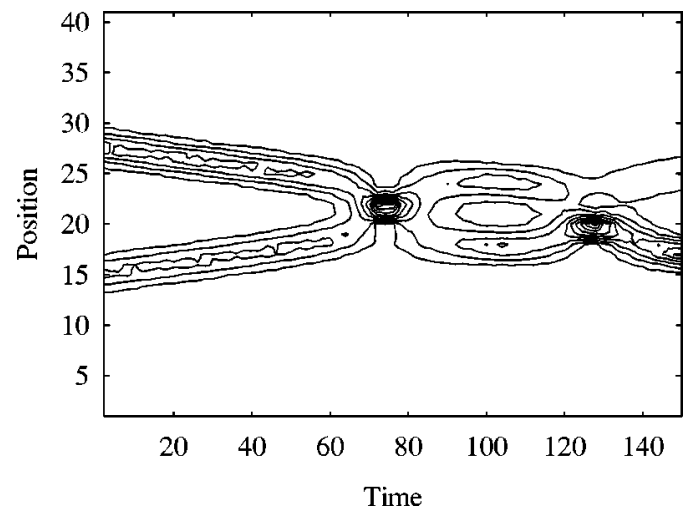

FIG. 10. An example of multiple collisions; $E_{1}=0.20, E_{2}$ $=0.25$. 

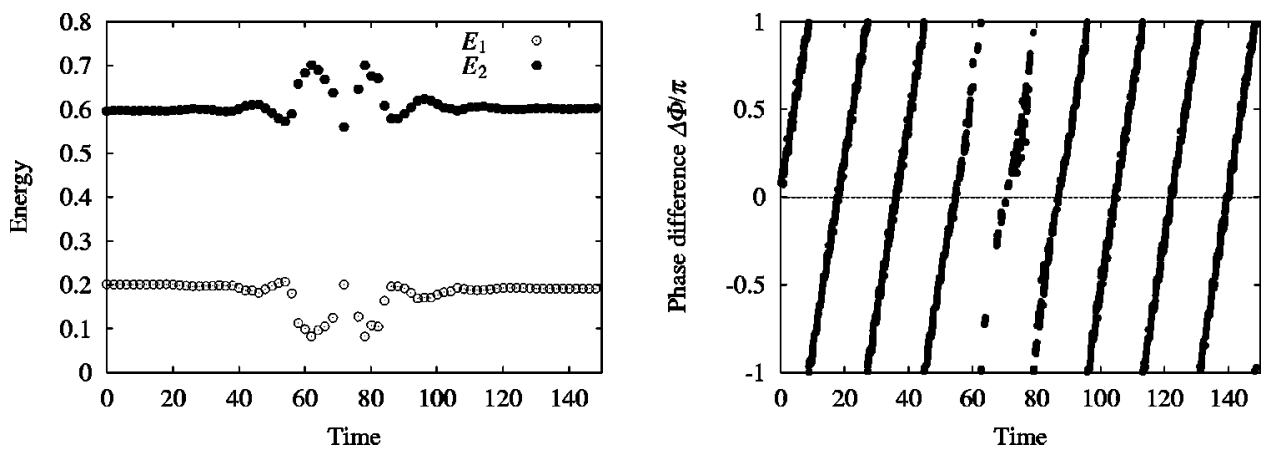

FIG. 11. Dynamics of two ILMs during collision. Same as Fig. 3 except for $E_{1}=0.20, E_{2}$ $=0.60$. region into two regions in view of interchange of ILM's energy: (b) interchange can take place and (c) not. These regions correspond to the cases due to the difference of ILM's energy as classified above in this section. If the energy difference of two ILMs before collision is relatively small, the transferred energy becomes so large that the magnitude of ILM's energy interchanges. On the other hand, if the energy difference is large before collision, the transferred energy is so small that interchanges do not occur. Transfer of large energy as in the small energy difference case takes place when the energy of ILMs becomes the same. Once the magnitudes of energy interchange, the energy transfer leads to widening of the energy difference of the two ILMs. These interchanges do not occur unless energies of ILMs become the same. Thus the boundary of the region (b) and (c) is determined by whether the two ILMs can transfer so large energy such that they become of the same energy or not.

This mechanism can be explained in view of the difference of ILM's angular frequency. In the case of small energy difference, the difference of angular frequency is also small. While the ILMs react with each other, the phase difference changes as time progresses due to the change of energy difference, but it is relatively small. This means that the change in the direction of energy transfer occurs so gradually that the ILMs have enough time to transfer large energy. In the case of large energy difference, on the other hand, the difference of angular frequency is large and this initial difference is dominant even in the collisional process. The states of advanced phase and delayed phase arise repeatedly in a short time, so that ILMs cannot transfer large energy in one cycle. This is the reason why ILMs cannot transfer large enough energy to cause the interchange of ILM's energy.

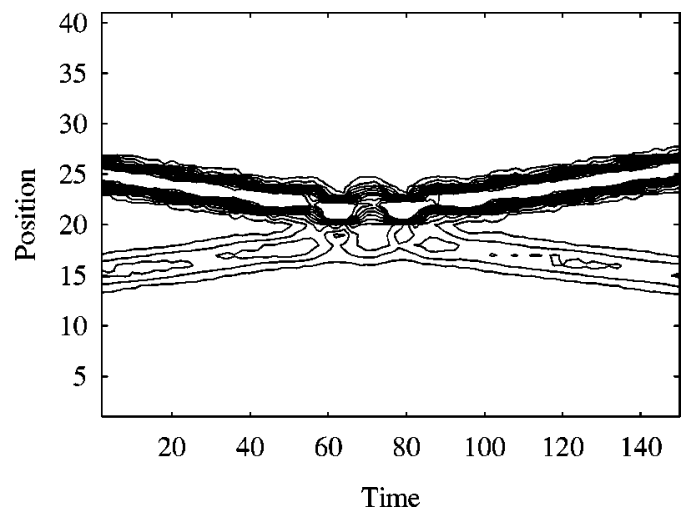

FIG. 12. Energy contour corresponding to Fig. 11.
Note that the results mentioned above are those for head-on collisions. The case of overtaking collisions is also considered and some differences from the case of head-on collisions are found: (i) ILMs interact for longer time than the case of head-on collisions, but (ii) transferred energy is much smaller for the same energy case $\left(E_{1}=E_{2}\right)$. These differences are caused by differences in relative velocity in the ILMs. Relative velocities in overtaking collisions are about 0.1 times of those in head-on collisions. The ILMs approach more slowly and do not approach near enough to interact strongly. Therefore we conclude that the overtaking collisions have lesser importance than the head-on collisions in view of the energy transfer.

\section{DISCUSSION}

\section{A. The mechanism of energy exchange}

Taking into account the results given in the preceding section, we propose a heuristic model so as to explain the energy transfer mechanism. In a previous paper [9], the model which describes collisions of solitons in perturbed NLS have been proposed. In this model, solitons are considered as particles which attract with potential as distance. We also propose a particle model extended to take the effect of the phase of ILMs into account.

Numerical results show that the transfer of energy in collision depends on the phase difference of two ILMs but not on the magnitude of energy. Figure 15 shows the time variation of $d E_{2} / d t$ and $\sin \Delta \Phi$. We can see that the ILM with advanced phase absorbs energy from another ILM. The rate

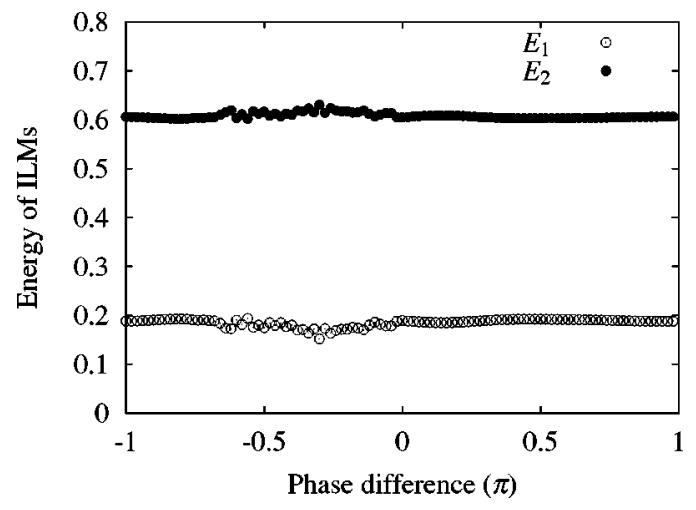

FIG. 13. Relation between the phase difference $\Delta \Phi$ at the instance of collision and the energies of ILMs after collision $\left(E_{1}^{\prime}\right.$ and $\left.E_{2}^{\prime}\right)$. Same as Fig. 5 except for $E_{1}=0.20, E_{2}=0.60$. 


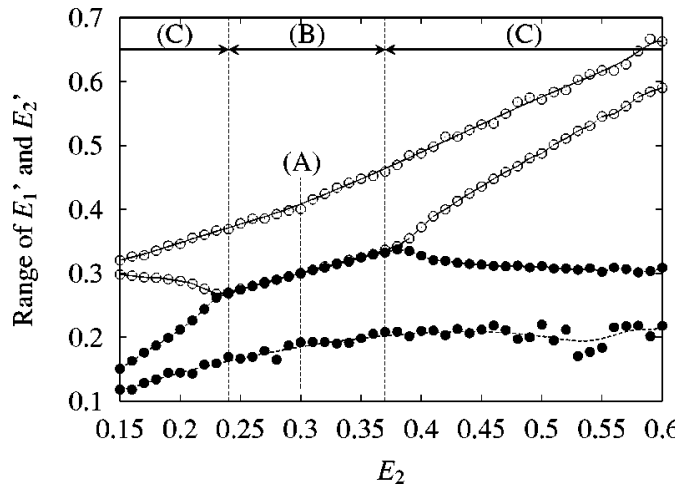

FIG. 14. Relation between the range of ILM's energy after collision and $E_{2}$ with fixed $E_{1}(=0.3)$. White circles indicate larger ILM and black indicate smaller one. Upper circles show maximum energy and lower ones show minimum energy. Labels (a)-(c) denote the energy difference of two ILMs corresponding to the classifications in Sec. IV: (a) same energy case, (b) small energy difference case, and (c) large energy difference case.

of the energy transfer $d E_{1,2} / d t$ becomes zero at $\Delta \Phi=\pi$, $-\pi$ and $d E_{1,2} / d t$ changes in like manner as $\sin \Delta \Phi$. The rate $d E_{1,2} / d t$ should be zero at $\Delta \Phi=0$ because of the continuity of $d E_{1,2} / d t$. Therefore we assume that the ratio of energy transfer is a function of $\Delta \Phi$, that is, $\sin \Delta \Phi$.

Figure 15 also implies that the energy transfer of ILMs occurs only when two ILMs approach enough to react and transferred energy becomes greater as the distance of ILMs becomes smaller. Therefore we assume that the rate of energy transfer also depends on the distance of the ILMs exponentially.

Adding to this, two ILMs at near enough distance are assumed to exert an attractive force on each other when they are almost in phase $(\Delta \Phi \simeq 0)$ and a repulsive one when they are out of phase $(\Delta \Phi \simeq \pi)$. Because the ILMs with $\Delta \Phi$ $=0$ fuse and those with $\Delta \Phi=\pi$ reflect without reaction in the same energy case, we assume that the force which works between the ILMs depends on the term $-\cos (\Delta \Phi)$ and on the distance exponentially.

Taking the above assumptions into account, we propose here a set of equations as follows:

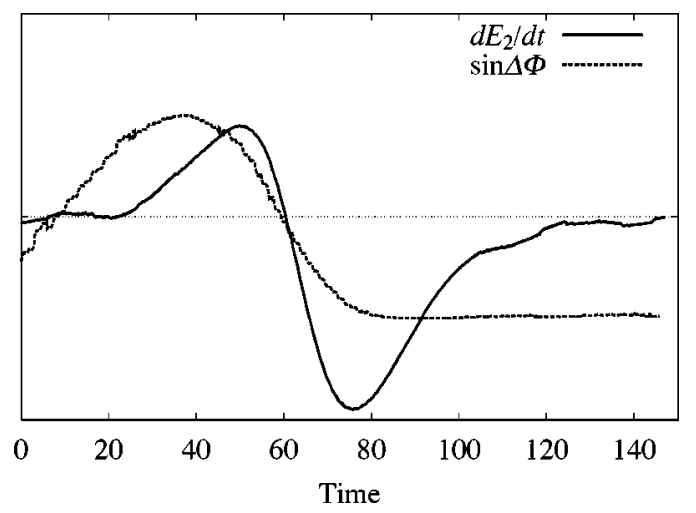

FIG. 15. Time evolution of $d E_{2} / d t$ (solid line) which corresponds to the case of Fig. 7(a). Dotted line represents the change of $\sin \Delta \Phi$ for comparison with $d E_{2} / d t$ (see details in Sec. V A).

$$
\begin{gathered}
\frac{d E_{1}}{d t}=-\frac{A}{2} \sin \Delta \Phi \exp \left(-\frac{\bar{x}}{L}\right), \\
\frac{d E_{2}}{d t}=\frac{A}{2} \sin \Delta \Phi \exp \left(-\frac{\bar{x}}{L}\right), \\
\frac{d \Delta \Phi}{d t}=F\left(E_{2}\right)-F\left(E_{1}\right), \\
\frac{d^{2} \bar{x}}{d t^{2}}=-B \cos \Delta \Phi \exp \left(-\frac{\bar{x}}{L}\right),
\end{gathered}
$$

where $\bar{x}$ denotes the distance between the two ILMs, and $A$, $B$, and $L$ are constants. $A$ denotes the strength expressing how the rate of energy transfer depends on the phase difference and the distance. $B$ denotes the same strength for the distance between two ILMs. $L$ is a standard length for nondimensionalization. $F(E)$ is the function which approximates the relation between the energy $E$ and the angular frequency $\omega$ of the ILM, and it is given by using a result of preliminary calculations shown in Fig. 1(a), as follows:

$$
F(E)=\sqrt{E}+1.73 .
$$

We solved these equations numerically for various conditions in comparison with the full dynamical simulations. The results shown in Figs. 16 and 17 agree qualitatively well with the results of the full dynamical simulations. Figure 18 shows the relation of the phase difference to the transferred energy corresponding to the case shown in Figs. 5, 9, and 13. Note that in Fig. 18 there is a range of the phase difference where the numerical results of Eqs. (13)-(16) do not provide correct results because the fusion of the ILMs occurs $(\bar{x}$ $=0)$ in such a range. In the full dynamical simulations, the results in that range are quite different from the others, showing something like chaotic behaviors.

\section{B. Random collisions in the chaotic breathers}

In terms of the properties found in this paper for the ILMs collision, we try to explain certain aspects of the chaotic breathers (CBs) [18]. It is observed that many ILMs generated by the modulational instability of the high frequency initial mode collide with each other and eventually come to form one big ILM which moves in the system in an erratic way and termed the $\mathrm{CB}$.

First, we consider the change of the energy between before and after collision. Dauxois and Peyrard [18] pointed out that the ILM with larger energy absorbs the energy on an average from other ILMs through the collisions. This tendency is also shown statistically in the Klein-Gordon lattices [17]. However when we pay attention to the detailed collision process, it does not match with the existing explanations in some cases. In the collision of moving ILMs, the magnitude of energy can be interchanged in the case where the energy difference of the ILMs before collision is relatively small [see Figs. 7(c) and (d)]. This inconsistency can be resolved if we focus only on the magnitude of energy $E_{1}, E_{2}$, 

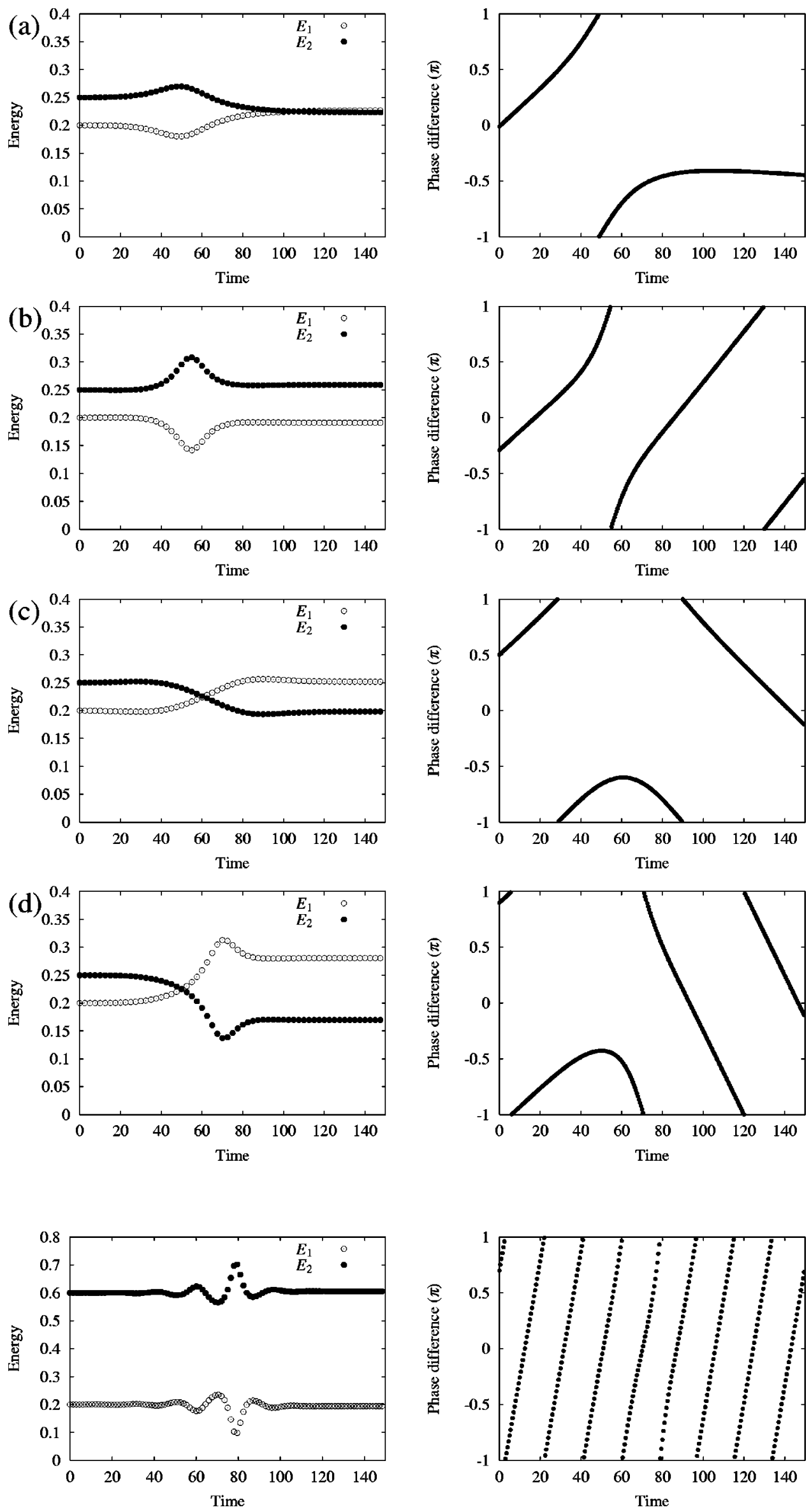

FIG. 16. Numerical results of heuristic model equations (13)(16) for the energy of ILMs and the phase difference. Each figure corresponds to four cases shown in Fig. $7\left(E_{1}=0.20, E_{2}=0.25\right)$.

FIG. 17. Same as Fig. 14 except for $E_{1}=0.20, E_{2}=0.60$. 

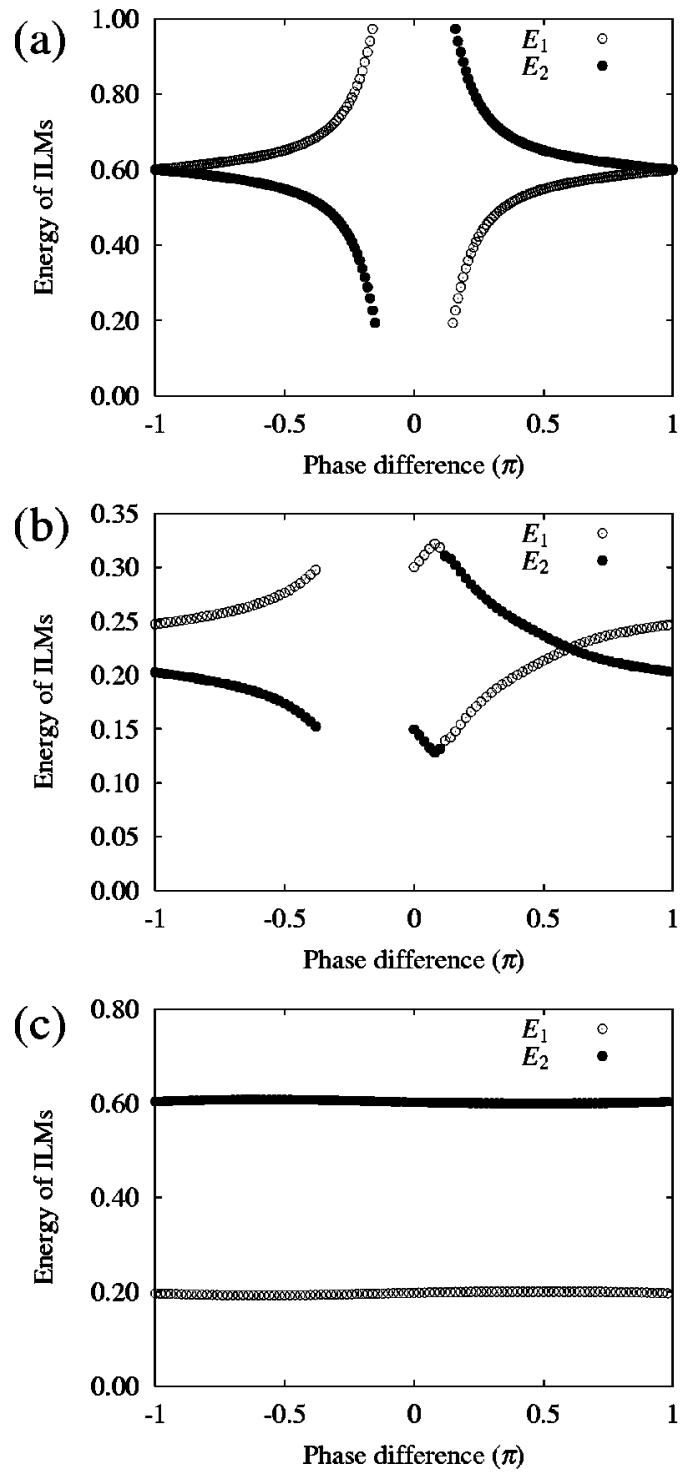

FIG. 18. Relation between the phase difference and the energy of ILMs after collision calculated by the model equations (13)(16): (a) $E_{1}=E_{2}=0.60 ;$ (b) $E_{1}=0.20, E_{2}=0.25$; and (c) $E_{1}$ $=0.20, E_{2}=0.60$. No points are shown in the region $[-0.2<\Delta \Phi$ $<0.2$ in (a) and $-0.4<\Delta \Phi<-0.05$ in (b)], because the model equations cannot describe the repeated collisions found in the full particle simulations.

without distinguishing the identity of modes, $M_{1}$ an $M_{2}$. From this point of view, we can say that the energy of the larger ILM after collision $\left[\max \left(E_{1}^{\prime}, E_{2}^{\prime}\right)\right]$ is larger than that before the collision $\left[\max \left(E_{1}, E_{2}\right)\right]$ in many cases. This can be a reason why only one breather grows in the formation of CBs. In addition, in the case where the energy difference is large before the collision, the energy transfer is small and the interchange of the magnitude of energy does not occur. Thus the larger ILM also remains larger after collision. In the view of exchange of energy, the larger ILM tends to absorb the energy from the smaller ILM in many cases not only of the results of numerical calculations but also of those of model equations.
Second, we see the relation of the energy transfer to the chaotic behavior of CBs. Energy transfers of the ILMs sometimes become singular due to the strong reaction of two ILMs. Chaotic scattering of solitons is also reported by Dmitriev et al. in the case of the perturbed NLS system [8-10]. They have reported irregular scattering of solitons occurring when the phase difference is nearly zero. There are some differences i.e., in our result, ILMs can collide inelastically in almost all phase differences (in the case of small energy difference), but in the perturbed NLS case, solitons collide elastically in a wide range of phase differences. But the dynamics of solitons with chaotic scattering is quite similar to the present case.

When two ILMs approach near enough and their phase difference becomes nearly zero, they affect to attract strongly with each other. As a result they are fused and separated again. While the ILMs go to fuse, the difference in the angular frequency becomes large because of large energy transfer to one side. This large difference in the angular frequency causes the next in-phase state within the distance near enough to react. Then subsequent collisions can occur. Especially, this mechanism can induce a bound state of ILMs [19] only when two ILMs have the same energy and mirror symmetry. In other cases the ILMs collide a few times due to the difference in symmetry. In repeated collisions, ILM's property changes rapidly as time progresses. The energy (and the other variables) after collision in the region showing random changes sensitively depends on the small deviations in the phase difference and energy of the two ILMs before and during collision. Adding to this, the difference in symmetry affects the behavior of the ILMs during repeated collisions. This random and sensitive behavior of the transferred energy may be a cause for the stochastic behavior of the ILMs.

\section{CONCLUDING REMARKS}

In this paper, we examined the collisions of the ILMs in the FPU $\beta$ lattice in detail, and we showed that the magnitude of the transferred energy depends crucially on the phase difference. Taking into account the numerical results of temporal changes of energy, position and phase of ILMs in the collisional process, we have proposed a simple set of equations that describes the inelastic collisions of the ILMs. The solutions of these equations could explain the numerical results qualitatively very well.

In some cases, the magnitude of the transferred energy sensitively depends on the small phase difference and symmetry, which leads to stochastic changes of the transferred energy. This is due to the repeated collisions which occur when the phase difference is close to zero and the distance between the ILMs is small enough to react. This behavior may have relevance to the stochastic motion of the CBs.

Our results of energy transfer are consistent with the statistical explanation in the previous papers. We can explain qualitatively the mechanisms of the growth of ILMs in CBs formation process in terms of the results in this paper. However, a quantitative comparison of the results of the full dynamical simulations of CBs with those of the model equations proposed in this paper is required as a next step in view 
of the stochastic process of multiple collisions. Adding to this, there remain some problems to be clarified further, that is,

(i) The behavior of ILMs in the crossover region between small and large energy difference and

(ii)detailed analysis of collisions in consideration of the exact collision point of ILMs. These topics are left for a future investigation.

\section{ACKNOWLEDGMENT}

This work was partially supported by a Sasakawa Scientific Research Grant from the Japan Science Society.
[1] A.J. Sievers and S. Takeno, Phys. Rev. Lett. 61, 970 (1988).

[2] S. Flach and C.R. Willis, Phys. Rep. 295, 181 (1998).

[3] R. Lai, and A.J. Sievers, Phys. Rev. Lett. 81, 1937 (1998).

[4] S.F. Mingaleev, Yu.S. Kivshar, and R.A. Sammut, Phys. Rev. E 62, 5777 (2000).

[5] K. Hori, and S. Takeno, J. Phys. Soc. Jpn. 61, 4263 (1992).

[6] Yu.S. Kivshar and D.K. Campbell, Phys. Rev. E 48, 3077 (1993).

[7] M.J. Ablowitz, Z.H. Musslimani, and G. Biondini, Phys. Rev. E 65, 026602 (2002).

[8] S.V. Dmitriev, D.A. Semagin, A.A. Sukhorukov, and T. Shigenari, Phys. Rev. E 66, 46609 (2002).

[9] S.V. Dmitriev and T. Shigenari, Chaos 12, 324 (2002).

[10] D.A. Semagin, S.V. Dmitriev, T. Shigenari, Y.S. Kivshar, and A. A Sukhorukov, Physica B 316-317, 136 (2002).
[11] D. Cai, A.R. Bishop, and N. Grønbech-Jensen, Phys. Rev. E 56, 7246 (1997).

[12] J.B. Page, Phys. Rev. B 41, 7835 (1990).

[13] T. Cretegny, T. Dauxois, S. Ruffo, and A. Torcini, Physica D 121, 109 (1998).

[14] V.V. Mirnov, A.J. Lichtenberg, and H. Guclu, Physica D 157, 251 (2001).

[15] K. Ullmann, A.J. Lichtenberg, and G. Corso, Phys. Rev. E 61, 2471 (2000).

[16] I. Daumont, T. Dauxois, and M. Peyrard, Nonlinearity 10, 617 (1997).

[17] O. Bang and M. Peyrard, Phys. Rev. E 53, 4143 (1996).

[18] T. Dauxois and M. Peyrard, Phys. Rev. Lett. 70, 3935 (1993).

[19] Yu.S. Kivshar, A.R. Champneys, D. Cai, and A.R. Bishop, Phys. Rev. B 58, 5423 (1998). 